\title{
HP1 $\beta$ suppresses metastasis of human cancer cells by decreasing the expression and activation of MMP2
}

\author{
SANG AH YI ${ }^{1}$, HYUN-WOOK RYU ${ }^{2}$, DONG HOON LEE ${ }^{2,3}$, JEUNG-WHAN HAN ${ }^{1}$ and SO HEE KWON ${ }^{2}$ \\ ${ }^{1}$ Research Center for Epigenome Regulation, School of Pharmacy, Sungkyunkwan University, Suwon, Gyeonggi 440-746; \\ ${ }^{2}$ College of Pharmacy, Yonsei Institute of Pharmaceutical Sciences, Yonsei University, Incheon 406-840; \\ ${ }^{3}$ Department of Integrated OMICS for Biomedical Science, Yonsei University, Seoul 120-749, Republic of Korea
}

Received July 7, 2014; Accepted August 26, 2014

DOI: $10.3892 /$ ijo.2014.2646

\begin{abstract}
Heterochromatin protein 1 (HP1) is an epigenetic modifier of gene regulation and chromatin packing via binding to trimethylated histone $\mathrm{H} 3$ lysine 9 (H3K9). HP1 plays an important role in gene activation as well as gene repression in heterochromatin and euchromatin. However, the role of individual HP1 proteins in human diseases remains elusive. Here, we show that HP1 $\beta$ negatively regulates the expression and activation of matrix metallopeptidase (MMP)2, which mediates cancer metastasis by destructing type IV collagen. Reduced HP1 $\beta$ expression correlates with the increased level of pro- and active-MMP2 in colon cancer cells. Consistently, HP1 $\beta$ knockdown (KD) increased and HP1 $\beta$ overexpression decreased the mRNA level of MMP2 and membrane type 1 metallopeptidase (MT1-MMP). Furthermore, cancer cells overexpressing HP1 $\beta$ showed impaired migratory ability, whereas HP1 $\beta$-deleted cancer cells had increased migration. HP1 $\beta$ negatively regulates MMP2 expression in a transcriptional level and prevents MMP2 activation through reducing the expression of MT1-MMP. These findings shed new light on HP1 $\beta$ as a molecular regulator and an efficient therapeutic target of metastatic cancer.
\end{abstract}

\section{Introduction}

Heterochromatin protein 1 (HP1) is a histone code reader which specifically recognizes and binds to methylated $\mathrm{H} 3$

Correspondence to: Dr So Hee Kwon, College of Pharmacy, Yonsei Institute of Pharmaceutical Sciences, Yonsei University, 162-1 Songdodong, Yeonsu-gu, Incheon 406-840, Republic of Korea

E-mail: soheekwon@yonsei.ac.kr

Abbreviations: ECM, extracellular matrix; H3K9me, histone $\mathrm{H} 3$ lysine 9 methylation; HP1, heterochromatin protein 1; KD, knockdown; MMP, matrix metallopeptidase; MT1-MMP, membrane type 1 matrix metallopeptidase; TIMP, tissue inhibitor of metallopeptidase

Key words: HP1 $\beta$, MMP2, MT1-MMP, TIMP2, migration, cancer metastasis lysine 9 (H3K9) (1). Through its activities in DNA and its interacting proteins, the mammalian HP1 family plays a critical role in a variety of cellular processes including centromere stability, telomere stability, regulation of gene expression, DNA repair, cellular senescence and cancer progression (2-4). Three human HP1 homologs (HP1 $\alpha$, HP1 $\beta$ and HP1 $\gamma$ ) contain two conserved domains, which are separated by a flexible hinge region: a chromodomain (CD) interacting with methylated H3K9 and a chromoshadow domain (CSD) interacting with the PxVxL motif of its partner (5). HP1 homologs are known to exhibit different subnuclear localizations in interphase: HP1 $\alpha$ and HP1 $\beta$ are centromeric while HP1 $\gamma$ is located in both euchromatic and heterochromatic regions $(3,6,7)$. As might be expected given their name and localization, HP1 in mice and human represses transcription and suppresses tumor through formation of multimolecular complex with transcriptional corepressor TIF1 $\beta$, histone methyltransferase SETDB1 and NuRD-histone deacetylase complex (8). However, it has been recently reported that HP1 may work at euchromatic regions and as a positive regulator in gene expression $(5,9,10)$.

Several reports show that expression of HP1 is changed in various tumor tissues compared with normal tissues. In papillary thyroid carcinomas and embryonal brain tumor, the mRNA level of HP1 $\alpha$ was decreased $(11,12)$, which makes HP1 $\alpha$ a predictor in these cancers. HP1 $\alpha$ expression is also decreased in invasive/metastatic breast cancer tissue and HP1 $\alpha$ knockdown (KD) reduces the invasion rate of metastatic breast cancer cells $(13,14)$, suggesting HP1 $\alpha$ is a metastatic suppressor. In addition to HP1 $\alpha$, reduction of HP1 $\beta$ expression is correlated with invasive activity in human melanoma cells (15). Different from these beneficial effects, the three homologs of HP1 were highly expressed in patients with acute myeloid leukemia and chronic myeloid leukemia (16). However, the molecular mechanism whereby the expression of HP1 proteins is regulated in cancer is not well understood.

Matrix metallopeptidases (MMPs) are a family of zinc-dependent extracellular matrix (ECM) remodeling endopeptidases capable of degrading almost all components of ECM (17). MMPs are important not only in normal, physiological and biological processes such as embryogenesis, normal tissue remodeling, wound healing and angiogenesis but also in diseases such as arthritis and cancer (18). Expression and activation of MMP are increased in almost all human cancers 

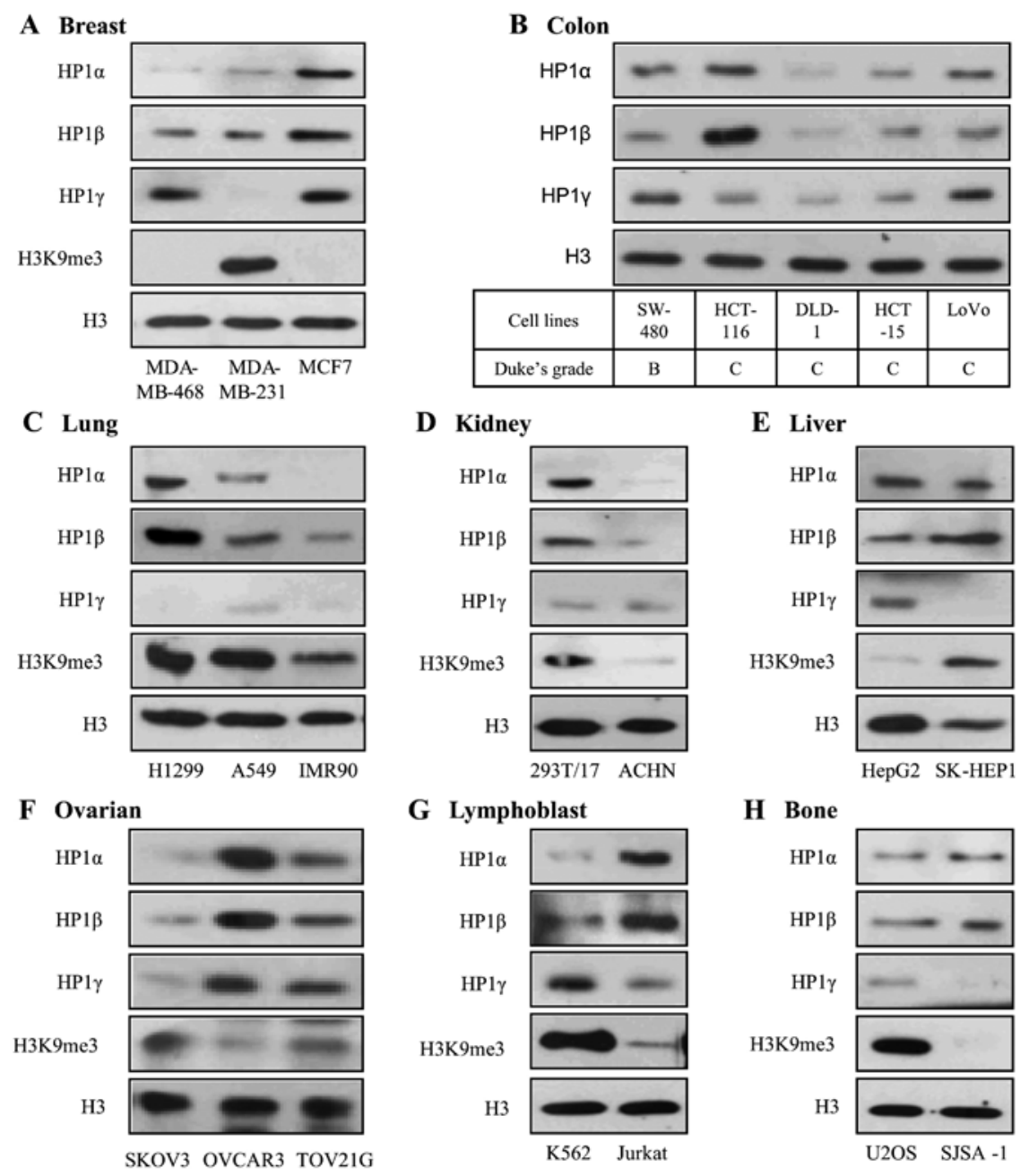

Figure 1. The expression level of heterochromatin protein 1 (HP1) proteins in diverse human cancer cell lines. HP1 detected by immunoblot analysis using whole cell protein lysates from (A) three breast cancer cell lines, (B) five colon cancer cell lines, (C) three lung cancer or normal cell lines, (D) two kidney cancer or normal cell lines, (E) two liver cancer cell lines, (F) three ovarian cancer cell lines, (G) two leukemia cell lines, and (H) two osteosarcoma cell lines.

compared to normal tissue. Indeed, MMPs play important roles in carcinogenesis as well as invasion and metastasis (19). MMPs can regulate cell migration by removing sites of adhesion, exposing new sites, cleaving cell-cell or cell-matrix receptors and releasing chemo attractants from ECM (20). MMP2/14 degrades laminin 5 and reveals a cryptic site that triggers motility (19). In addition to migration, MMP2/9 are localized in invadopodia and promote invasion by degrading type IV collagen of basement membrane $(21,22)$.

To understand this issue, we have identified the expression patterns of HP1 in various cancer cells. We show that HP $1 \beta$ inhibits de novo expression and activation of MMP2 through the control of mRNA level of MMP2 and membrane type 1 metallopeptidase (MT1-MMP). Collectively, this study is the first to elucidate the functional relationship of HP $1 \beta$ and MMP2 in cancer metastasis.

\section{Materials and methods}

Cell culture and transfection. Human cancer cell lines were cultured according to the instructions from ATCC and were maintained under a fully humidified atmosphere of $95 \%$ air and $5 \% \mathrm{CO}_{2}$ at $37^{\circ} \mathrm{C}$. Cancer cells were grown to $70 \%$ confluence in plates, the cells were transfected with siRNA or GFP-tagged vectors using Lipofectamine 2000 reagent, according to manufacturer's protocol (Invitrogen Life Technologies, Carlsbad, CA, USA). After incubation for $48 \mathrm{~h}$, mRNAs and proteins were extracted from the cells. The sequences of siRNAs targeting HP1 genes were: HP1 $\alpha$, 5'-GUUCCAGUCCUCUCUCAAAGC-3' and 5'-GCUUUG AGAGAGGACUGGAAC-3'; HP1 $\beta$, 5'-GACUCCAGUGGA GAGCUCAUG-3' and 5'-CAUGAGCUCUCCACUGGA GUC-3'; HP1 $\gamma, 5$ '-AUUCUUCAGGCUCUGCCUC-3' and 5'-GAGGCAGAGCCUGAAGAAU-3' (HP1 $\alpha \mathrm{HP} 1 \beta$, and HP1 $\gamma$ primers were described previously) (23).

Western blotting. Immunoblotting was performed as previously described (24). Cell lysates were prepared using lysis buffer supplemented with protease and phosphatase inhibitors (Thermo Scientific Pierce, Rockford, IL, USA). Protein concentrations were quantified according to the BCA Protein Assay kit (Thermo Scientific Pierce, Rockford, IL, USA).

RT-PCR and quantitative real-time PCR (qRT-PCR). Total RNA was extracted using easy-Blue reagent (Intron 
Table I. The human cancer cell lines investigated.

\begin{tabular}{|c|c|c|c|c|c|}
\hline Organ & Cell line & Tumor type/stage & $\mathrm{HP} 1 \alpha$ & $\mathrm{HP} 1 \beta$ & $\mathrm{HP} 1 \gamma$ \\
\hline \multirow[t]{3}{*}{ Breast } & MDA-MB-468 & Malignant adenocarcinoma & -- & - & + \\
\hline & MDA-MB-231 & Malignant adenocarcinoma & -- & - & -- \\
\hline & MCF7 & Adenocarcinoma & + & + & + \\
\hline \multirow[t]{5}{*}{ Colon } & SW-480 & Colorectal adenocarcinoma/Duke's grade B & + & + & + \\
\hline & HCT-116 & Colorectal carcinoma/Duke's grade C & + & ++ & - \\
\hline & DLD-1 & Colorectal adenocarcinoma/Duke's grade C & -- & -- & -- \\
\hline & HCT-15 & Colorectal adenocarcinoma/Duke's grade C & - & - & - \\
\hline & LoVo & Colorectal adenocarcinoma/Duke's grade C & - & - & + \\
\hline \multirow[t]{3}{*}{ Lung } & H1299 & Carcinoma & + & ++ & -- \\
\hline & A549 & Carcinoma & - & + & - \\
\hline & IMR90 & Normal fibroblast & -- & - & -- \\
\hline \multirow[t]{2}{*}{ Kidney } & $293 \mathrm{~T} / 17$ & Normal epithelial cell & + & + & - \\
\hline & $\mathrm{ACHN}$ & Renal cell adenocarcinoma & -- & -- & - \\
\hline \multirow[t]{2}{*}{ Liver } & HepG2 & Hepatocellular carcinoma & + & - & + \\
\hline & SK-HEP1 & Adenocarcinoma & - & + & - \\
\hline \multirow[t]{3}{*}{ Ovarian } & SKOV3 & Adenocarcinoma & - & - & - \\
\hline & OVCAR3 & Adenocarcinoma & ++ & ++ & ++ \\
\hline & TOV21G & Malignant adenocarcinoma/grade 3, Stage III & + & + & + \\
\hline \multirow[t]{2}{*}{ Lymphoblast } & K562 & Chronic myelogenous leukemia & -- & - & + \\
\hline & Jurkat & Acute T cell leukemia & + & ++ & - \\
\hline \multirow[t]{2}{*}{ Bone } & $\mathrm{U} 2 \mathrm{OS}$ & Osteosarcoma & - & - & - \\
\hline & SJSA-1 & Multipotential osteosarcoma & + & + & -- \\
\hline
\end{tabular}

Biotechnology, Seoul, Korea) and $1 \mu \mathrm{g}$ of RNA with oligo dT primers was subjected to reverse transcription using the ImProm-II ${ }^{\mathrm{TM}}$ Reverse Transcription system (Promega Corporation, Madison, WI, USA). cDNA was amplified using Super Premix Sapphire PCR master mix (Mbiotech, Inc., Seoul, Korea). qRT-PCR was performed with the KAPA ${ }^{\mathrm{TM}}$ SYBR ${ }^{\circledR}$ FAST qPCR (Kapa Biosystems, Inc., Wilmington, MA, USA) using CFX96 ${ }^{\mathrm{TM}}$ or $\mathrm{Chromo}^{\mathrm{TM}}$ real-time PCR Detector (Bio-Rad, Hercules, CA, USA). Relative levels of mRNA were normalized to the values of GAPDH mRNA for each reaction. Primer sequences used for PCR were: MMP2, 5'-ACCAGCTGGCCTAGTGATGATG-3' and 5'-GGCTT CCGCATGGTCTCGATG-3'; MT1-MMP, 5'-GGAATAAC CAAGTGATGGATGG-3' and 5'-TTGTTTCCACGGAAGA AGTAGG-3'; tissue inhibitor of metallopeptidase (TIMP)2, 5'-GCGGTCAGTGAGAAGGAAGTGG-3' and 5'-CTTGCA CTCGCAGCCCATCTG-3'; GAPDH, 5'-GAGTCAACGGAT TTGGTCGT-3' and 5'-TTGATTTTGGAGGGATCTCG-3'.

Migration assay. Human cancer cells grown in 6-well plates were scratched with a yellow pipette tip to form a thin wound. Microscope images were observed immediately and $48 \mathrm{~h}$ after the scratch.

\section{Results}

Analysis of HPl expression in human cancer cell lines. To investigate the potential role of HP1 in human cancer, we first examined the expression levels of the three HP1 proteins in diverse human cancer cell lines, which originated from eight different organs. The cell lines we used are indicated in Table I. HP1 $\alpha$ and HP1 $\beta$ were more highly expressed in MCF7, a non-invasive breast cancer cell line, than the two invasive breast cancer cell lines (Fig. 1A). HP1 $\gamma$ was uniquely repressed and histone $\mathrm{H} 3$ lysine 9 methylation (H3K9me3) was dramatically increased in only MDA-MB-231, invasive breast cancer cells. Colon cancer cells are sorted according to the Dukes grade. Among the five colon cancer cell lines, only SW-480 is classified as Dukes grade B, which has invasion through the bowel wall, but does not involve lymph nodes. The expression levels of HP1 $\alpha, \beta$ and $\gamma$ were all high in SW-480 (Fig. 1B). The other colon cancer cell lines, which are classified as Dukes grade $\mathrm{C}$, involving invasion of the lymph nodes, expressed high levels of all three HP1 proteins. In IMR90, a normal lung cell line, the three HP1 proteins and $\mathrm{H} 3 \mathrm{~K} 9 \mathrm{me} 3$ were all suppressed compared to the two lung cancer cell lines (Fig. 1C). On the other hand, the HP1 proteins and H3K9me3 were more highly expressed in 293T/17, normal kidney cell line, than ACHN, renal cell adenocarcinoma (Fig. 1D). These results suggest that HP1 proteins are related to tumor progression in lung, but tumor suppression in kidney. In the liver cancer cell lines, HP1 $\alpha$ and HP1 $\gamma$ were more highly expressed in HepG 2 while HP1 $\beta$ and H3K9me3 were more highly expressed in SK-HEP1 (Fig. 1E). In the ovarian cancer cell lines, the three HP1 proteins showed similar expression patterns, but H3K9me3 showed 
$\mathbf{A}$

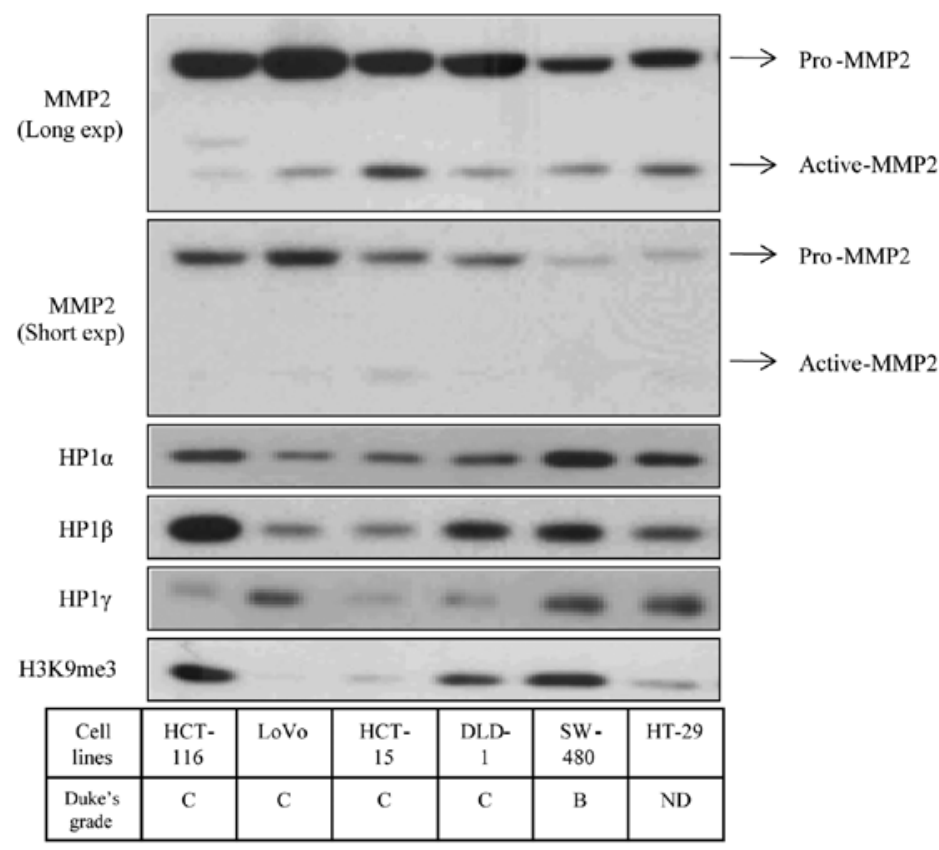

B

HP1a-active-MMP2
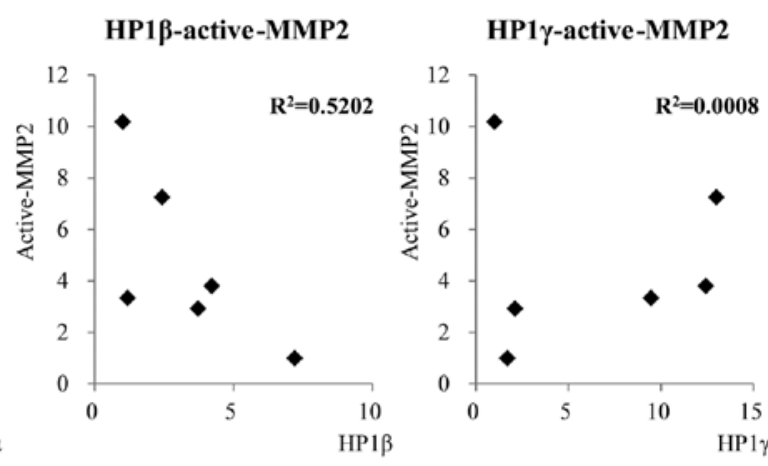

Figure 2. The reverse expression level of matrix metallopeptidase (MMP) 2 and heterochromatin protein 1 (HP1) $\beta$ in colon cancer cell lines. (A) Immunoblot analysis of whole cell lysates from six colon cancer cell lines. (B) The correlation graphs of quantified expression level of HP1s and active-MMP2 measured.

an opposite pattern (Fig. 1F). In leukemia and osteosarcoma cell lines, HP1 $\gamma$ and H3K9me3 were expressed in the same pattern (Fig. 1G and $\mathrm{H}$ ). Taken together, these results show that the expression patterns of the HP1 proteins vary in different cancer types and degree of invasiveness (Table I).

Inverse correlation of $M M P 2$ and $H P 1 \beta$ in colon cancer cells. Although to different extents, the three HP1 proteins were highly expressed in colon cancer cell lines. To determine whether HP1 and MMP2 are involved in colorectal cancer, we first analyzed the expression pattern of MMP2 and HP1 proteins in six colon cancer cell lines. MMP2, which degrades the basic component of cellular membrane, is regarded as a leading molecule in the metastatic process of cancer development $(17,19)$. Inactive pro-MMP2 is $70 \mathrm{kDa}$ and active-MMP2, derived from cleavage of pro-MMP2 is smaller (35-50 kDa). Among the members of HP1 family, HP1 $\beta$ showed a reverse expression pattern with active-MMP2 (Fig. 2A). Using ImageJ program, we quantified the expression level of active-MMP2 and HP1s displayed in Fig. 2A and assessed the correlation between the values. We confirmed a strong correlation between active-MMP 2 and HP $1 \beta$ through the coefficient of determination $\left(\mathrm{R}^{2}=0.5202\right)$ (Fig. 2B, middle). HP1 $\alpha$ showed a weak correlation with active-MMP2, as $\mathrm{R}^{2}$ is 0.1066 (Fig. 2B, left) and HP1 $\gamma$ had no correlation with active-MMP2 (Fig. 2B, right). These data suggest the possibility of a biological relationship between HP1 $\beta$ and MMP2.

$H P 1 \beta$ negatively regulates the expression and activation of $M M P 2$ protein. To investigate whether HP1 $\beta$ has influence on the MMP2 protein level, we transfected GFP-empty vector or GFP-tagged HP1 vectors into colon cancer cell line, HCT-15, which showed a weak HP1 $\beta$ signal. Overexpression of HP1 $\beta$ in HCT-15 cells resulted in reduction of pro-MMP2 as well as active-MMP2 (Fig. 3A). In OVCAR3, an ovarian cancer cell line, overexpressing HP1 $\beta$ caused a reduction of pro-MMP2, but not active-MMP2 (Fig. 3B). Similarly, only the pro-MMP2 level in HeLa cells was decreased depending on the amounts of ectopically expressed HP1 $\beta$ (Fig. 3C). We cannot exclude the possibility that, because the high basal expressions of HP1 $\beta$ is enough to saturate the active-MMP2 level, exogenously expressed HP1 $\beta$ results in no significant change in the active-MMP2 level in OVCAR3 and HeLa cells (Fig. 3E and F). Therefore, we next examined the MMP2 level in HP1 $\beta$-depleted cells. In HT-29, a colon cancer cell line, KD of HP1 $\beta$ promoted 

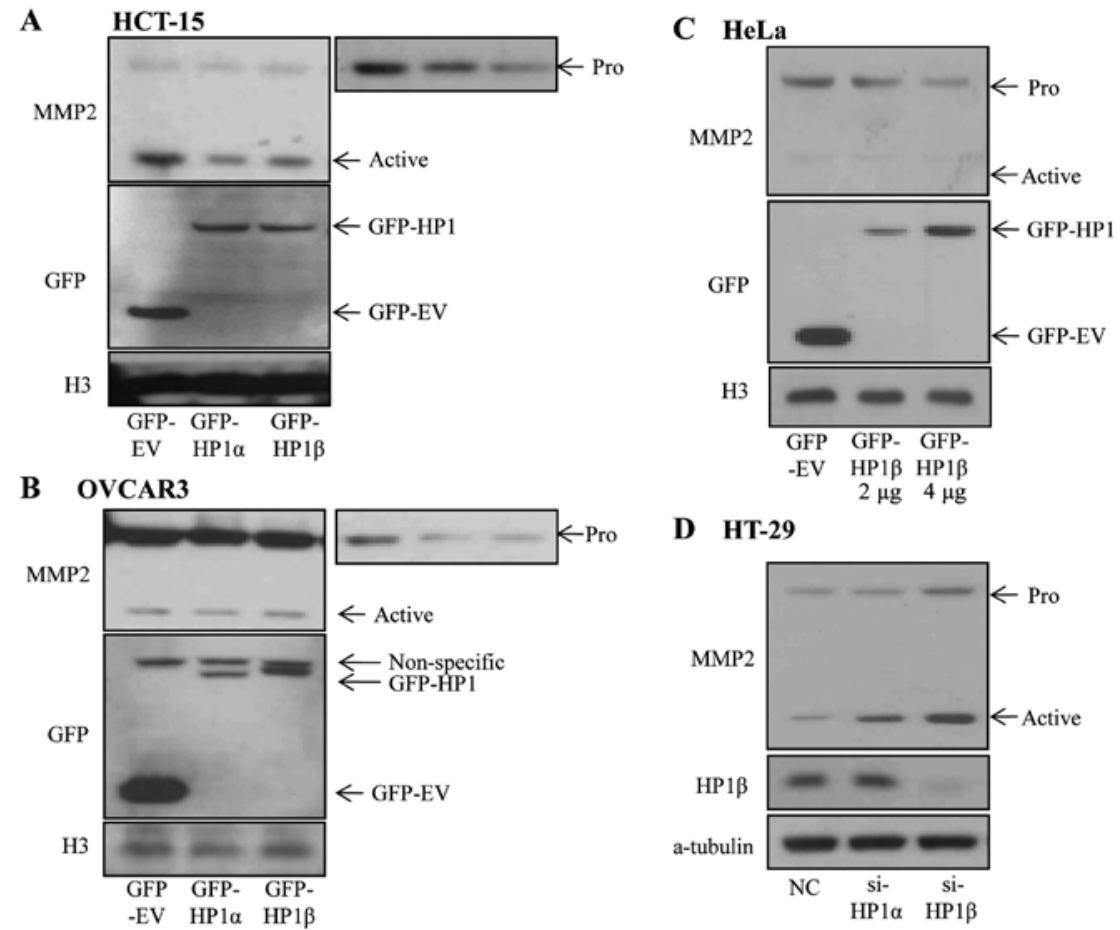

D HT-29
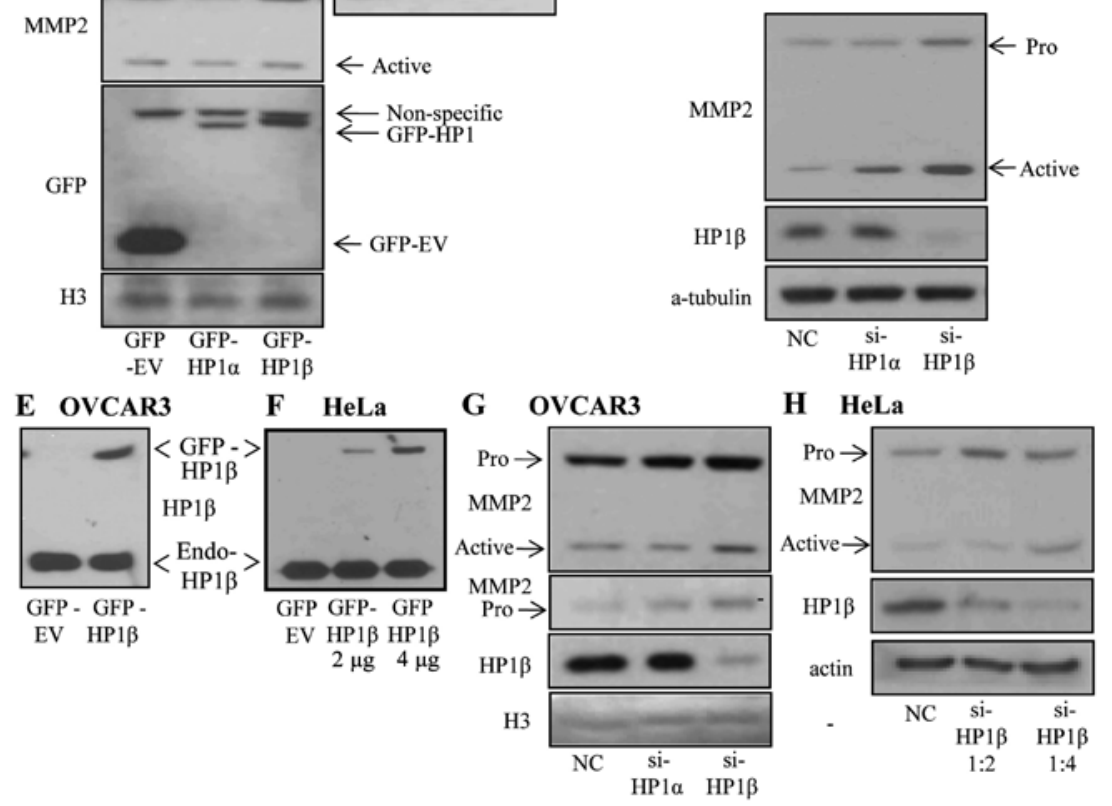

Figure 3. The expression and activation of matrix metallopeptidase (MMP)2 protein is negatively regulated by heterochromatin protein 1 (HP1) $\beta$. Immunoblot analysis of whole cell lysates from (A) HCT-15, (B and E) OVCAR3 and (C and F) HeLa cells transfected with $2 \mu$ g of GFP EV, GFP-tagged HP1 $\alpha$ or HP1 $\beta$ vectors. Immunoblot analysis of whole cell lysates from (D) HT29, (G) OVCAR3 and (H) HeLa cells transfected with control si-RNA, HP1 $\alpha$ or HP1 $\beta$ siRNA using different ratios of Lipofectamine (1:2 or 1:4).

both pro- and active-MMP2 (Fig. 3D). Increase of pro-MMP2 and active-MMP2 levels following HP1 $\beta$ KD, was observed in OVCAR3 and HeLa cells (Fig. 3G and H). These effects were also observed in HP1 $\alpha$ but to a lesser extent (Fig. 3A-D and G). Thus, these results suggest that HP1 $\beta$ is a major regulator of MMP2 expression and activation and HP1 $\alpha$ is a minor regulator.

$H P 1 \beta$ regulates the $m R N A$ expression of $M M P 2$ and $M T 1-M M P$. To examine whether HP1 $\beta$ regulates MMP2 expression at the transcription level, we measured the mRNA level of MMP2 in HP1 $\beta$-deleted cells. The mRNA level of the MMP2 gene increased 1.5 -fold, following KD of HP1 $\beta$ in HCT-15 cells (Fig. 4A). However, considering that HCT-15 cells express very low basal level of HP1 $\beta$, this is regarded as a significant change. In OVCAR3 and HeLa cells, which express a high basal level of HP1 $\beta$, the mRNA level of MMP2 increased $>5$-fold following KD of HP1 $\beta$ (Fig. 4B and C).

The activation of MMP2 is triggered by the cleavage of pro-MMP2 by MT1-MMP, which is influenced by the level of TIMP. TIMP2 binds to the active site of MT1-MMP and this complex acts as a receptor for pro-MMP2. Then, the free
MT1-MMP cleaves the propeptide of pro-MMP2, leading to the intermediate stage. Through further autocatalytic proteolysis, fully active MMP2 is generated (Fig. 5A) (25). However, when the TIMP2 level is high enough to saturate all MT1-MMP, cleavage of pro-MMP2 is impossible preventing MMP2 activation (Fig. 5B). We found that active-MMP2 as well as pro-MMP2 was changed by HP1 $\beta$ (Figs. 3 and 4 ). Thus, we examined whether the mRNA levels of MT1-MMP and TIMP 2 are regulated by HP1 $\beta$. The mRNA levels of MMP2 and MT1-MMP decreased following overexpression of HP1 $\beta$ in HCT-15 and OVCAR 3 cells, while the level of TIMP2 did not significantly change (Fig. 5C and D). Consistent with this result, KD of HP1 $\beta$ promotes the mRNA level of MMP2 and MT1-MMP in both cell lines (Fig. 5E and F).

HPl $\beta$ inhibits migration of human cancer cells. Recent studies of breast cancer cells revealed an inverse correlation between HP1 $\alpha$ expression and tumor cell invasiveness $(13,14)$. We therefore investigated whether tumor cell motility is regulated by all three HP1 protein or in an HP1 isoform-specific manner. To do this, we assessed the migratory ability of HeLa 


\section{A $\quad$ HCT-15}

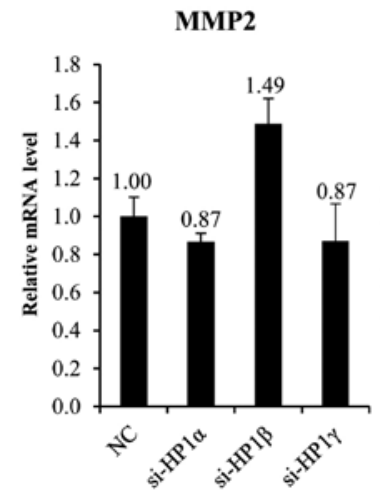

B OVCAR3

MMP2

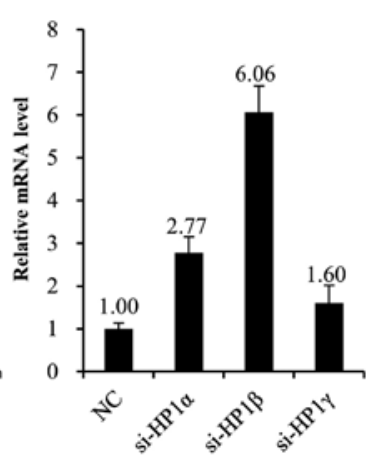

C HeLa

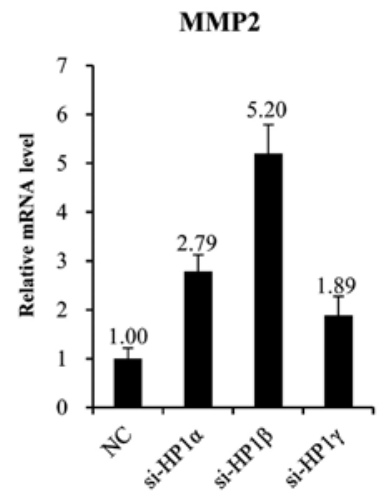

Figure 4. Knockdown (KD) of heterochromatin protein 1 (HP1) $\beta$ increases the mRNA level of matrix metallopeptidase (MMP)2. The mRNA levels of MMP2 gene in (A) HCT-15, (B) OVCAR3 and (C) HeLa cells transfected with control si-RNA or HP1 $\alpha, \beta$, or $\gamma$ siRNA were measured by real-time PCR. The mRNA levels were normalized to the mRNA level of GAPDH. Error bars represent SD $(n=3)$.

A Low TIMP-2

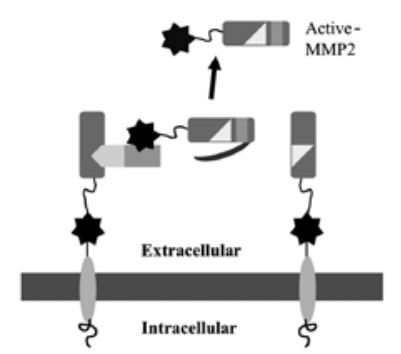

B High TIMP-2

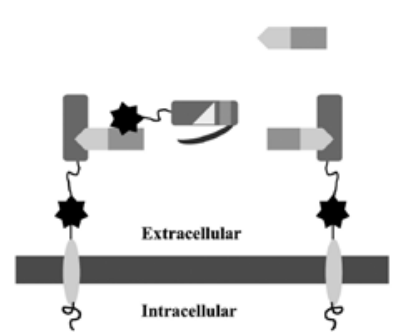

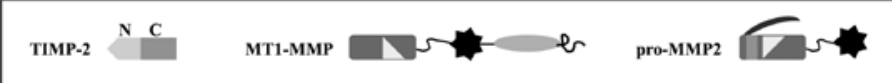

C $\quad$ HCT-15

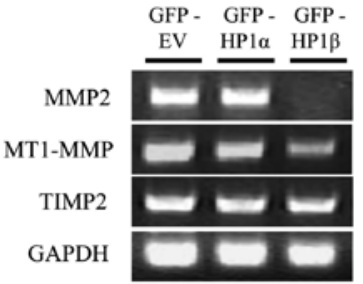

E $\quad$ HCT-15

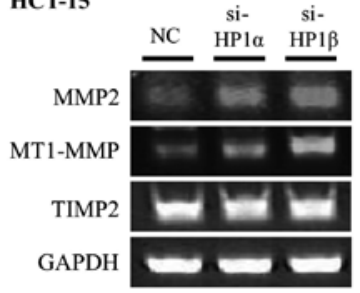

D OVCAR3

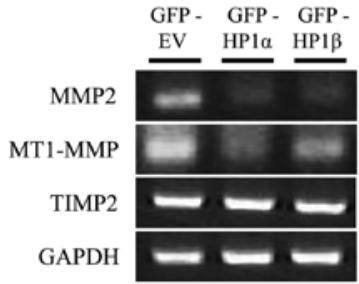

F OVCAR3

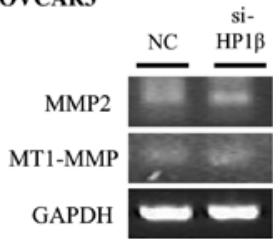

Figure 5. Heterochromatin protein 1 (HP1) $\beta$ reduces the mRNA levels of matrix metallopeptidase (MMP)2 and membrane type 1 metallopeptidase (MT1-MMP). (A) The activation mechanism of pro-MMP2 by MT1-MMP when the tissue inhibitor of metallopeptidase (TIMP)2 level is low. (B) Disturbed activation of pro-MMP2 when the TIMP2 level is high. The mRNA levels of MMP2, MT1-MMP, TIMP2 and GAPDH genes in (C) HCT-15 and (D) OVCAR3 cells transfected with GFP-EV or GFP-tagged HP1 $\alpha$, or $\beta$ vectors were measured by RT-PCR. (E) The mRNA levels of MMP2, MT1-MMP, TIMP2 and GAPDH genes in HCT-15 cells transfected with control si-RNA or HP1 $\alpha$ or $-\beta$ siRNA were measured by RT-PCR. (F) The mRNA levels of MMP2, MT1-MMP and GAPDH genes in OVCAR3 cells transfected with control si-RNA or HP1 $\beta$ siRNA were measured by RT-PCR.

cells transfected with GFP-tagged vectors or siRNA following scratch with a yellow tip. Overexpression of HP1 $\beta$ markedly decreased the migration rate of HeLa cells (Fig. 6A), while
KD of HP1 $\beta$ promoted migration of HeLa cells (Fig. 6B). Interestingly, overexpression or KD of HP1 $\alpha$ resulted in the similar, but lesser effects, on the migration rate compared to 
A

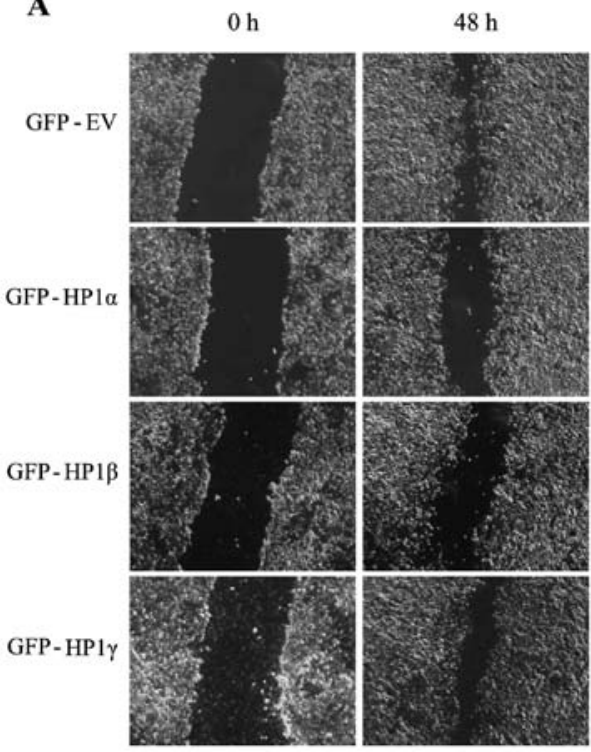

B



Figure 6. Heterochromatin protein 1 (HP1) $\beta$ prevents migration of human cancer cells. (A) HeLa cells were transfected with $4 \mu \mathrm{g}$ of GFP-EV or GFP-tagged HP1 $\alpha, \beta$, or $\gamma$ vectors using Lipofectamine. After $6 \mathrm{~h}$, the cells were scratched with a yellow tip. After $48 \mathrm{~h}$, the migration rate of cells was observed with a microscope. (B) HeLa cells were transfected with control si-RNA or HP1 $\alpha, \beta$, or $\gamma$ siRNA using Lipofectamine. After $24 \mathrm{~h}$, the cells were scratched with a yellow tip. After $48 \mathrm{~h}$, the migration rate of cells was observed with a microscope.

HP1 $\beta$. In contrast, modulating the expression of HP1 $\gamma$ did not affect the migration. Thus, it suggests that HP1 $\beta$ and HP1 $\alpha$, but not HP1 $\gamma$, are responsible for cancer metastasis.

\section{Discussion}

The HP1 family of proteins was identified $>25$ years ago (26), but the common or divergent functions of the three homologs remain unclear. There are several indications that the three mammalian HP1 homologs, HP1 $\alpha, \beta$ and $\gamma$, may not fulfil identical functions. First, they show differences in cellular distribution. HP1 $\alpha$ marks strongly in the pericentric heterochromatin, whereas HP1 $\gamma$ shows less specificity for these regions $(3,6,7)$. Second, despite their high similarity in structure and function, the three HP1 homologs are not always present together and can interact with different binding partners (3). Finally, distinct post-translational modifications on an individual HP1 homolog $(6,27)$ may further diversify their functions. Here, we show that in human cells, HP1 $\beta$ has unique properties in cancer development, which are not shared by HP1 $\alpha$ and $\gamma$. First, we find opposite expression patterns of HP1 $\beta$ and MMP2, which promotes metastasis in cancer. Second, we reveal that overexpression of HP1 $\beta$ reduces protein levels of both pro-MMP2 and active-MMP2; this is mediated by suppressed mRNA level of MMP2 and MT1-MMP, which are required for the activation of MMP2. Consistently, KD of HP1 $\beta$ increases protein levels of pro-MMP 2 and active-MMP2. mRNA level of MMP2 and MT1-MMP are also increased by HP1 $\beta$ KD. Furthermore, we demonstrate that overexpression of HP1 $\beta$ represses the migration of human cancer cell and KD of HP1 $\beta$ promotes cell migration.

The links between HP1 proteins and tumorigenesis have emerged. In vitro studies of HP1 expression in cancer have suggested that these proteins protect against tumor cell aggressiveness and invasiveness. HP1 $\alpha$ is overexpressed in carcinomas and the expression level correlates with clinical data and disease outcome (28). This has been most convincingly shown in breast cancer cell lines $(13,14)$. Downregulation of the HP1 $\alpha$ protein is linked to a higher invasive potential of cancer cells $(3,11,13,29)$. HP1 $\alpha$ downregulation has been observed in most highly invasive and metastatic breast cancer cells versus non-metastatic cells $(13,14,29)$. Decreasing the HP1 $\alpha$ expression in non-invasive MCF-7 cells enhanced the invasive potential; increasing the HP1 $\alpha$ expression in metastatic MDA-MB-231 cells decreased the invasive potential (30). In this study, we show that HP1 $\beta$ overexpression causes impaired migratory ability, whereas HP1 $\beta$ KD results in increased migration. Moreover, our findings reveal the molecular mechanism by which HP1 $\beta$ regulates cancer migration and metastasis. HP1 $\beta$ negatively regulates MMP2 expression at the transcriptional level and prevents MMP2 activation by reducing the expression of MT1-MMP. In line with this finding, decreased HP1 $\beta$ expression is associated with melanoma oncogenesis and high invasiveness in human melanoma cells (15). Consequently, because HP1 $\alpha$ and HP1 $\beta$ have been shown to attenuate metastasis in cancer cells, it may be, given the data presented here, that HP1 is a suppressor of cell migration and metastasis. Interestingly, the reverse pattern of euchromatic HP1 $\gamma$ and H3K9me3 was observed in different cancer cells including breast, ovarian, lung, and liver cancer cells (Fig. 1). This result supports the hypothesis that HP1 $\gamma$ might regulate certain cancer-associated genes via a different epigenetic mechanism, not shared by HP1 $\alpha$ and $\beta$.

Taken together, our results elucidate the role of HP1 $\beta$ as a key regulator of cancer metastasis by reducing both expression and activation of MMP2, which is mediated by altered mRNA levels of MMP2 and MT1-MMP. These findings suggest the epigenetic regulation of MMP2 by HP1 $\beta$ and provide the mechanistic rationale for the targeting of HP1 $\beta$ to relieve cancer metastasis. 


\section{Acknowledgements}

This research was supported by Medical Research Center programs (2012-0009849) and the Basic Science Research Program through the National Research Foundation of Korea (NRF) funded by the Ministry of Education, Science and Technology (2012013998).

\section{References}

1. Nielsen PR, Nietlispach D, Mott HR, et al: Structure of the HP1 chromodomain bound to histone $\mathrm{H} 3$ methylated at lysine 9. Nature 416: 103-107, 2002.

2. Ayoub N, Jeyasekharan AD and Venkitaraman AR: Mobilization and recruitment of HP1: a bimodal response to DNA breakage. Cell Cycle 8: 2945-2950, 2009.

3. Dialynas GK, Vitalini MW and Wallrath LL: Linking Heterochromatin Protein 1 (HP1) to cancer progression. Mutat Res 647: 13-20, 2008

4. Zhang R and Adams PD: Heterochromatin and its relationship to cell senescence and cancer therapy. Cell Cycle 6: 784-789, 2007.

5. Kwon SH and Workman JL: The changing faces of HP1: from heterochromatin formation and gene silencing to euchromatic gene expression: HP1 acts as a positive regulator of transcription. Bioessays 33: 280-289, 2011.

6. Minc E, Allory Y, Worman HJ, Courvalin JC and Buendia B: Localization and phosphorylation of HP1 proteins during the cell cycle in mammalian cells. Chromosoma 108: 220-234, 1999.

7. Nielsen AL, Oulad-Abdelghani M, Ortiz JA, Remboutsika E, Chambon $\mathrm{P}$ and Losson R: Heterochromatin formation in mammalian cells: interaction between histones and HP1 proteins. Mol Cell 7: 729-739, 2001.

8. Nielsen AL, Ortiz JA, You J, et al: Interaction with members of the heterochromatin protein 1 (HP1) family and histone deacetylation are differentially involved in transcriptional silencing by members of the TIF1 family. EMBO J 18: 6385-6395, 1999.

9. Hediger $F$ and Gasser SM: Heterochromatin protein 1: don't judge the book by its cover! Curr Opin Genet Dev 16: 143-150, 2006.

10. Piacentini L, Fanti L, Berloco M, Perrini B and Pimpinelli S: Heterochromatin protein 1 (HP1) is associated with induced gene expression in Drosophila euchromatin. J Cell Biol 161: 707-714, 2003.

11. Pomeroy SL, Tamayo P, Gaasenbeek M, et al: Prediction of central nervous system embryonal tumour outcome based on gene expression. Nature 415: 436-442, 2002.

12. Wasenius VM, Hemmer S, Kettunen E, Knuutila S, Franssila K and Joensuu $\mathrm{H}$ : Hepatocyte growth factor receptor, matrix metalloproteinase-11, tissue inhibitor of metalloproteinase-1, and fibronectin are up-regulated in papillary thyroid carcinoma: a cDNA and tissue microarray study. Clin Cancer Res 9: 68-75, 2003.

13. KirschmannDA,LiningerRA,GardnerLM, et al: Down-regulation of HP1Hsalpha expression is associated with the metastatic phenotype in breast cancer. Cancer Res 60: 3359-3363, 2000.
14. Thomsen R, Christensen DB, Rosborg S, Linnet TE, Blechingberg J and Nielsen AL: Analysis of HPl $\alpha$ regulation in human breast cancer cells. Mole Carcinog 50: 601-613, 2011.

15. Nishimura K, Hirokawa YS, Mizutani $\mathrm{H}$ and Shiraishi T: Reduced heterochromatin protein 1-beta (HP1beta) expression is correlated with increased invasive activity in human melanoma cells. Anticancer Res 26: 4349-4356, 2006.

16. Lukásová E, Koristek Z, Falk M, et al: Methylation of histones in myeloid leukemias as a potential marker of granulocyte abnormalities. J Leukoc Biol 77: 100-111, 2005.

17. Vihinen P and Kähäri VM: Matrix metalloproteinases in cancer: prognostic markers and therapeutic targets. Int J Cancer 99: 157-166, 2002

18. Gupta A, Kaur CD, Jangdey $M$ and Saraf S: Matrix metalloproteinase enzymes and their naturally derived inhibitors: novel targets in photocarcinoma therapy. Ageing Res Rev 13: 65-74, 2014.

19. Egeblad M and Werb Z: New functions for the matrix metalloproteinases in cancer progression. Nat Rev Cancer 2: 161-174, 2002

20. McCawley LJ and Matrisian LM: Matrix metalloproteinases: they're not just for matrix anymore! Curr Opin Cell Biol 13: 534-540, 2001.

21. Ikebe T, Shinohara M, Takeuchi $\mathrm{H}$, et al: Gelatinolytic activity of matrix metalloproteinase in tumor tissues correlates with the invasiveness of oral cancer. Clin Exp Metastasis 17: 315-323, 1999.

22. Thomas GT, Lewis MP and Speight PM: Matrix metalloproteinases and oral cancer. Oral Oncol 35: 227-233, 1999.

23. du Chéné I, Basyuk E, Lin YL, et al: Suv39H1 and HP1gamma are responsible for chromatin-mediated HIV-1 transcriptional silencing and post-integration latency. EMBO J 26: 424-435, 2007.

24. Kwon S, Zhang Y and Matthias P: The deacetylase HDAC6 is a novel critical component of stress granules involved in the stress response. Genes Dev 21: 3381-3394, 2007.

25. Lafleur MA, Handsley MM and Edwards DR: Metalloproteinases and their inhibitors in angiogenesis. Expert Rew Mol Med 5: 1-39, 2003.

26. James TC and Elgin SC: Identification of a nonhistone chromosomal protein associated with heterochromatin in Drosophila melanogaster and its gene. Mol Cell Biol 6: 3862-3872, 1986.

27. Lomberk G, Bensi D, Fernandez-Zapico ME and Urrutia R: Evidence for the existence of an HP1-mediated subcode within the histone code. Nat Cell Biol 8: 407-415, 2006.

28. De Koning L, Savignoni A, Boumendil C, et al: Heterochromatin protein 1alpha: a hallmark of cell proliferation relevant to clinical oncology. EMBO Mol Med 1: 178-191, 2009.

29. De Lange R, Burtscher H, Jarsch $M$ and Weidle UH: Identification of metastasis-associated genes by transcriptional profiling of metastatic versus non-metastatic colon cancer cell lines. Anticancer Res 21: 2329-2339, 2001.

30. Norwood LE, Moss TJ, Margaryan NV, et al: A requirement for dimerization of HP1Hsalpha in suppression of breast cancer invasion. J Biol Chem 281: 18668-18676, 2006. 